\title{
Cerebral Venous Sinus Thrombosis (CVST) in a Young Female with COVID-19 Infection and Oral Contraceptive Pill Ingestion Double Hit? - A Case Report
}

\author{
Apurva Dubey ${ }^{1}$, Sourya Acharya², Samarth Shukla ${ }^{3}$, Sunil Kumar ${ }^{4}$ \\ 1, 2, 4 Department of Medicine, Datta Meghe Institute of Medical Sciences (Deemed to be University), \\ Sawangi, Wardha, Maharashtra, India. ${ }^{3}$ Department of Pathology, Datta Meghe Institute of Medical \\ Sciences (Deemed to be University), Sawangi, Wardha, Maharashtra, India.
}

\section{INTRODUCTION}

The Chinese centre for disease control and prevention detected a novel severe acute respiratory syndrome - coronavirus 2 (SARS - CoV2) from a nasopharyngeal swab in a patient with atypical pneumonia in Wuhan, Hubei province, China on January, 2020. ${ }^{1}$ Corona virus disease-19 (COVID-19) has a high mortality rate in critically ill patients. Acute heart injury, acute kidney injury and sudden thromboembolic events are becoming more common and they can occur regardless of pulmonary or respiratory symptoms. ${ }^{1-5}$ COVID-19 has been shown to have the ability to create a hypercoagulable state in recent studies. ${ }^{2,3,6,7}$ Viral infections can cause endothelial cell dysfunction, resulting in excessive throbbing production and fibrinolysis inhibition. ${ }^{8-}$ 10 Hypoxia is also linked to an increase in blood viscosity and the activation of hypoxia-related genes that regulate coagulation and fibrinolysis making thrombotic events more likely.11,12 This septic-like coagulopathy can also lead to venous thrombosis, pulmonary embolism, and, in the worst-case scenario, disseminated intravascular coagulation. 3,4 Cerebral venous thrombosis, in particular, can manifest itself in a wide range of neurologic signs and symptoms. ${ }^{13,14}$ and had consistently fatal results.

\section{PRESENTATION OF CASE}

A 21-year-old female was admitted to us with chief complaints of fever for 3 days, headache with nausea and vomiting for 2 days and weakness of left side of body for 1 day. There was no history of cough, rhinorrhoea, breathlessness, haemoptysis, seizures and diplopia. She was on norethisterone $5 \mathrm{mg}$ for 5 months for amenorrhoea.

On admission, she was conscious, oriented (GCS - 14). General physical examination was normal. Oxygen saturation was $98 \%$ while breathing in ambient air. Central nervous system examination revealed intact cranial nerves, a complete left hemiplegia (power 0/5 in left upper and lower limbs). Cardiovascular and respiratory system were normal on examination. In view of fever, a rapid antigen test was performed for Covid-19, which was strongly positive. A nasopharyngeal swab was positive for SARS CoV- 2 infection.

Investigations revealed; haemoglobin - $13.3 \mathrm{gm} / \mathrm{dl}$, total leukocyte count (TLC) $5500 / \mathrm{mm}^{3}$, platelet count $3.9 \mathrm{lakh} / \mathrm{mm}^{3}$. C reactive protein (CRP), d - Dimer, ferritin, lactate dehydrogenase (LDH), homocysteine levels were within normal limits.

High resolution computed tomography (HRCT) of thorax revealed patchy areas of consolidation in inferior lingular segment of left upper lobe (CT severity score 01/25) suggestive of mild disease. (Figure 1) Magnetic resonance imaging (MRI) brain with MR-venogram revealed features of multiple venous sinus thrombosis. (Figure 2, 3A, B)
Corresponding Author: Dr. Sourya Acharya, Professor and Head, Department of Medicine, Datta Meghe Institute of Medical Sciences (Deemed to be University), Sawangi, Wardha Maharashtra, India. E-mail: souryaacharya74@gmail.com

DOI: $10.14260 / \mathrm{jemds} / 2021 / 623$

How to Cite This Article:

Dubey A, Acharya S, Shukla S, et al. Cerebral venous sinus thrombosis (CVST) in a young female with COVID-19 infection and oral contraceptive pill ingestion double hit? - a case report. J Evolution Med Dent Sci 2021;10(35):3060-3062, $10.14260 / \mathrm{jemds} / 2021 / 623$

Submission 21-04-2021, Peer Review 29-06-2021, Acceptance 07-07-2021, Published 30-08-2021.

Copyright (C) 2021 Apurva Dubey et al. This is an open access article distributed under Creative Commons Attribution License [Attribution 4.0 International (CC BY 4.0)] 

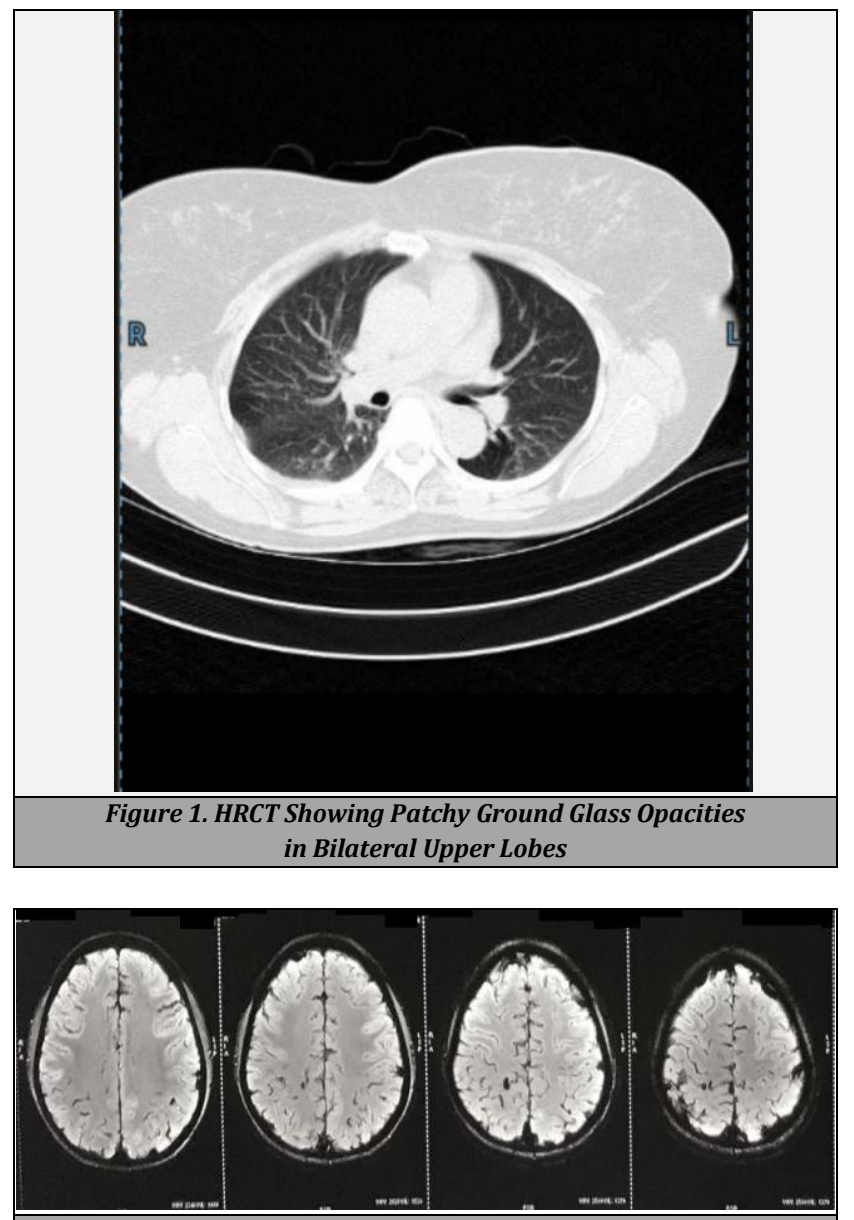

Figure 2. MRI Brain - There is Evidence of Loss of Normal Flow Void on T2 with Corresponding Loss of Flow Related Signal on Venogram in Region of Superior Sagittal Sinus, Left Transverse and Sigmoid Sinus with Associated Areas of Blooming on Gradient Recalled Echo Suggestive of Dural Venous Sinus Thrombosis

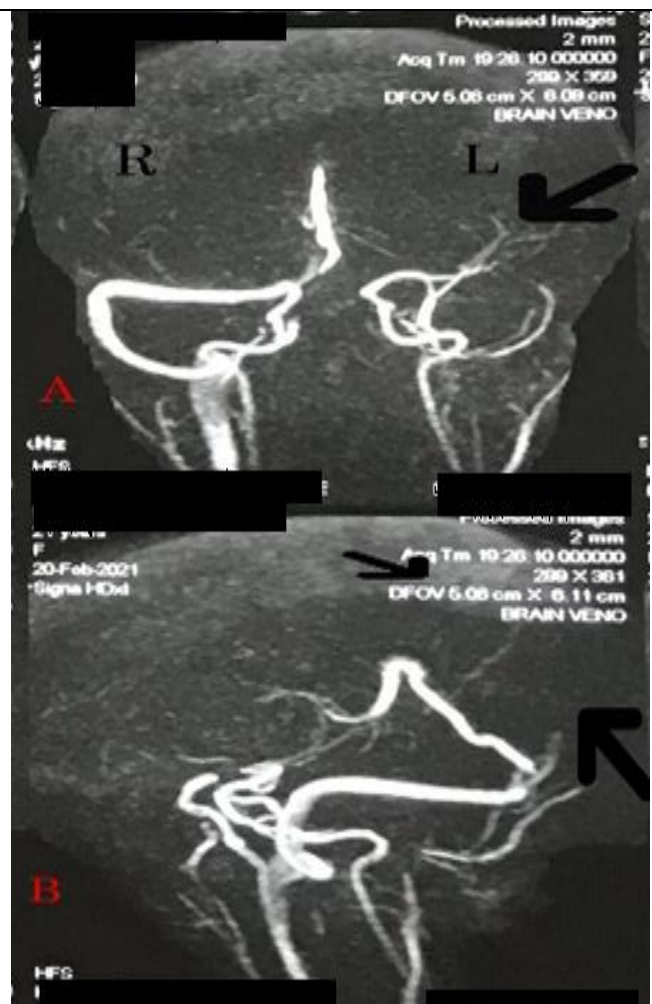

Figure 3.A; Coronal Section of MRV Showing Non-Visualization of Left Transverse and Sigmoid Sinus (Black Arrow); B: Sagittal Section Showing Non-Visualization of Superior Sagittal Sinus (Black Arrows)
There are associated areas of altered signal intensity in right parietal post central cortical region appearing intense on T1AI, heterogeneously hyperintense on T2/FLAIR, showing no restrictions on diffusion weighted imaging (DWI) suggestive of cortical venous infarcts with surrounding perilesional oedema.

Tab. norethisterone was stopped. She was treated with Tab. fabipiravir, Tab. doxycycline, multivitamins, Tab. levetiracetam $500 \mathrm{mg}$ BID, Inj. enoxaparin $0.6 \mathrm{mg}$ BID which was overlapped with Tab. warfarin $5 \mathrm{mg}$ to a target international normalized ratio (INR) of 2.5. She was discharged after 4 days and was advised to continue levetiracetam and warfarin and was asked for follow up INR measurements. On first follow up after 2 weeks power had significantly improved to $4 / 5$ on left side.

\section{DISCUSSION}

We describe a case of catastrophic cerebral venous thrombosis in young, previously healthy patient infected with SARS-CoV2, who were previously healthy and had no antecedent cardiopulmonary symptoms or fever. COVID-19 was most likely an additive risk factor in this series, as oral contraceptives is a risk factor in this case. Cerebral venous thrombosis (CVT) is relatively an uncommon cause of stroke, accounting for about 0.5 percent of all stroke cases and is having a confirmed mortality rate of $4 \%$.

CVT is caused by variety of causes, including oral contraceptives, pregnancy and puerperium, both of which trigger transient prothrombotic states. ${ }^{15,16} \mathrm{CVT}$ is responsible for $2 \%$ of pregnancy related strokes. ${ }^{17}$ Other major factors include diabetes mellitus, systemic inflammatory disease, cancer, hematologic disorders, head and neck infections, and hyperhomocysteinaemia and the patients were not routinely evaluated for any of these conditions.11,12,18,19 Another recognized risk factor for CVT is anaemia, which was not present in our patient. ${ }^{20}$ Coagulopathy is linked to a variety of viral infections. $8,10,21,22$ Viruses that activate endothelial cells directly or indirectly can impair coagulation and fibrinolytic systems.

Antiphospholipid antibodies have also been found in immunocompetent patients with many viral infections, including cytomegalovirus, human immunodeficiency virus (HIV), and varicella zoster virus.8,10 Patients with human herpes viruses (DNA viruses) and HIV are believed to be hypercoagulable.8,10 A ten-fold increased risk of venous thrombosis has been linked to chronic HIV infection. ${ }^{8}$

A recent research comparing the values of D-dimer, fibrin/ fibrinogen-degradation products, and fibrinogen in 94 patients with SARS-CoV-2 infection in a hospital in Wuhan with 40 healthy volunteers found that patients with COVID-19 had significantly higher values of D-dimer, fibrin/fibrinogen degradation products and fibrinogen. ${ }^{6}$ Wang et al. ${ }^{3}$ found positive associations between the degree of D-dimer elevation and the incidence of deep venous thrombosis with the severity of disease in 88 laboratory confirmed cases in Wuhan. This may be due to disproportionate endothelial cell activation.3,23

Oral contraception containing oestrogen or progesterone has been linked to rise in venous thromboembolism. The oestrogen dosage appears to be linked to venous thromboembolism, and the oestrogen and progesterone 
elements appear to be linked to arterial complications. The two conditions of blood coagulation and vascular thrombosis are inextricably linked. Oral contraception containing oestrogen/progesterone affects blood clotting by raising plasma fibrinogen and the activity of coagulation factors, especially factors VII and X; antithrombin III, a coagulation inhibitor, is typically reduced. Acceleration of aggregation also boosts platelet activity. Hypercoagulability is created as a result of these modifications, which tends to be partially offset by increased fibrinolytic activity. The coagulation effects of oral contraceptives in use today are dependent on the oestrogen dosage and the form of progesterone used in combination, according to studies.

\section{CONCLUSIONS}

This case report highlights the possible combined effects of oral contraceptive pills and superadded COVID-19 infection related cerebral venous thrombosis. There is evidence that COVID-19 and oral contraceptive pill use is significant contributor to hypercoagulation, raising the incidence of thrombosis. It is recommended that use of oral contraceptive pill in young females should be under supervision when superimposed COVID-19 infection ensues.

Financial or other competing interests: None.

Disclosure forms provided by the authors are available with the full text of this article at jemds.com.

\section{REFERENCES}

[1] Chen N, Zhou M, Dong X, et al. Epidemiological and clinical characteristics of 99 cases of 2019 novel coronavirus pneumonia in Wuhan, China: a descriptive study. Lancet 2020;395(10223):507-13.

[2] Li Y, Wang M, Zhou Y, et al. Acute cerebrovascular disease following COVID - 19: a single center, retrospective, observational study. SSRN Electronic Journal 2020;10.2139 / ssrn.3550025.

[3] Wang W, Sun Q, Bao Y, et al. Analysis of risk factors for the thromboembolic events from 88 patients with COVID - 19 Pneumonia in Wuhan, China: a retrospective descriptive report.

$(3 / 20 / 2020)$.

https://ssrn.com/abstract=3559633

or http://dx.doi.org/10.2139/ssrn.3559633

[4] Xie Y, Wang X, Yang P, et al. COVID-19 complicated by acute pulmonary embolism. Radiol Cardiothoracic Imaging 2020;2(2):e200067.

[5] Tang N, Li D, Wang X, et al. Abnormal coagulation parameters are associated with poor prognosis in patients with novel coronavirus pneumonia. J Thromb Haemost 2020;18(4):844-7.
[6] Han H, Yang L, Liu R, et al. Prominent changes in blood coagulation of patients with SARS - CoV - 2 infection. Clin Chem Lab Med 2020;58(7):1116-20.

[7] Zhang Y, Xiao M, Zhang S, et al. Coagulopathy and antiphospholipid antibodies in patients with Covid-19. N Engl J Med 2020;382(17):e38.

[8] Bibas M, Biava G, Antinori A. HIV-associated venous thromboembolism. Mediterr J Hematol Infect Dis 2011;3(1):e2011030.

[9] Squizzato A, Gerdes VEA, Buller HR. Effects of human cytomegalovirus infection on the coagulation system. Thromb Haemost 2005;93(3):403-10.

[10] Uthman IW, Gharavi AE. Viral infections and antiphospholipid antibodies. Semin Arthritis Rheum 2002;31(4):256-63.

[11] Gupta N, Zhao YY, Evans CE. The stimulation of thrombosis by hypoxia. Thromb Res 2019;181:77-83.

[12] Ortel TL. Acquired thrombotic risk factors in the critical care setting. Crit Care Med 2010;38(Suppl 2):S43-50.

[13] Canhao P, Ferro JM, Lindgren AG, et al. ISCVT Investigators. causes and predictors of death in cerebral venous thrombosis. Stroke 2005;36(8):1720-5.

[14] Girot M, Ferro JM, Canhao P, et al. Predictors of outcome in patients with cerebral venous thrombosis and intracerebral hemorrhage. Stroke 2007;38(2):337-42.

[15] Saposnik G, Barinagarrementeria F, Brown RD Jr, et al. Diagnosis and management of cerebral venous thrombosis: a statement for healthcare professionals from the American Heart Association / American Stroke Association. Stroke 2011;42(4):1158-92.

[16] Devasagayam S, Wyatt B, Leyden J, et al. Cerebral venous sinus thrombosis incidence is higher than previously thought: a retrospective population - based study. Stroke 2016;47(9):2180-2.

[17] James AH, Bushnell CD, Jamison MG, et al. Incidence and risk factors for stroke in pregnancy and the puerperium. Obstet Gynecol 2005;106(3):509-16.

[18] Carr ME. Diabetes mellitus: a hypercoagulable state. J Diabetes Complicate 2001;15(1):44-54.

[19] Martinelli I, Battaglioli T, Pedotti P, et al. Hyperhomocysteinemia in cerebral vein thrombosis. Blood 2003;102(4):1363-6.

[20] Coutinho JM, Zuurbier SM, Gaartman AE, et al. Association between anemia and cerebral venous thrombosis: case control study. Stroke 2015;46(10):2735-40.

[21] Goeijenbier M, van Wissen M, van de Weg C, et al. Review: viral infections and mechanisms of thrombosis and bleeding. J Med Virol 2012;84(10):1680-96.

[22] Netravathi M, Jaychandran R, Bhat M, et al. Profile of 26 HIV seropositive individuals with cerebral venous thrombosis. J Neurol Sci 2017;378:69-74.

[23] Yang J, Zheng Y, Gou X, et al. Prevalence of comorbidities in the novel Wuhan coronavirus (COVID - 19) infection: a systematic review and meta-analysis. Int J Infect Dis 2020;94:91-5. 